\title{
A Search for High-Mass Stars Forming in Isolation using CORNISH and ATLASGAL
}

\author{
Chenoa D. Tremblay ${ }^{1,5}$, Andrew J. Walsh ${ }^{1}$, Steven N. Longmore ${ }^{2}$, James S. Urquhart ${ }^{3,4}$ and Carsten König $^{3}$ \\ ${ }^{1}$ International Centre for Radio Astronomy Research, Curtin University, GPO Box U1987, Perth WA 6845, Australia \\ ${ }^{2}$ Astrophysics Research Institute, Liverpool John Moores University, Twelve Quays House, Egerton Wharf, Birkenhead CH41 1LD, UK \\ ${ }^{3}$ Max-Planck-Institut für Radioastronomie, Auf dem Hügel 69, Bonn, Germany \\ ${ }^{4}$ Centre for Astrophysics and Planetary Science, University of Kent, Canterbury, CT2 7NH, UK \\ ${ }^{5}$ Email: chenoa.tremblay@ postgrad.curtin.edu.au
}

(Received July 29, 2015; ACCEPTEd November 5, 2015)

\begin{abstract}
Theoretical models of high-mass star formation lie between two extreme scenarios. At one extreme, all the mass comes from an initially gravitationally bound core. At the other extreme, the majority of the mass comes from cluster scale gas, which lies far outside the initial core boundary. One way to unambiguously show high-mass stars can assemble their gas through the former route would be to find a high-mass star forming in isolation. Making use of recently available CORNISH and ATLASGAL Galactic plane survey data, we develop sample selection criteria to try and find such an object. From an initial list of approximately 200 sources, we identify the high-mass star-forming region G13.384 + 0.064 as the most promising candidate. The region contains a strong radio continuum source, that is powered by an early B-type star. The bolometric luminosity, derived from infrared measurements, is consistent with this. However, sub-millimetre continuum emission, measured in ATLASGAL, as well as dense gas tracers, such as $\mathrm{HCO}^{+}(3-2)$ and $\mathrm{N}_{2} \mathrm{H}^{+}(3-2)$ indicate that there is less than $\sim 100 \mathrm{M}_{\odot}$ of material surrounding this star. We conclude that this region is indeed a promising candidate for a high-mass star forming in isolation.
\end{abstract}

Keywords: stars: formation, stars: massive, (ISM): HII regions

\section{INTRODUCTION}

High-mass stars - $O$ or early $B$ type star of sufficient mass to produce a Type II supernova (Zinnecker \& York 2007) or $>8 \mathrm{M}_{\odot}$ (Miettinen 2012)_dominate the energy cycles and chemical enrichment of galaxies. However, understanding the formation of high-mass stars remains a challenge, and several different theoretical formation scenarios have been proposed (Zinnecker \& York 2007; Tan et al. 2014). The general observational phases (i.e., formation of cold dense cloud, gravitational collapse of a hot core, accretion, and formation of ultra-compact HII regions) are typically agreed upon, but the dominant physical processes and their relevant time scales are still under debate. The problems associated with our understanding are that it is difficult to observe the early stages of formation due to high dust extinction, the theoretical problem is complex and high-mass stars are seldom, if ever, formed in isolation (Zinnecker \& York 2007).

Over the last few decades, high-mass star formation theories have been discussed in the context of two extreme scenarios: that of the turbulent core (McKee \& Tan 2003) and the competitive accretion (Bonnell et al. 2001; Bonnell \& Bate 2006) scenarios. In the former, all the mass comes from an initially gravitationally-bound core. In the latter, the majority of the mass comes from cluster scale gas that is far outside the initial core boundary. Early debate suggested that stellar collisions (Bonnell, Bate, \& Zinnecker 1998) could be a potential creation mechanism, but this has largely been discounted due to the extremely high stellar densities required ${ }^{1}$. More recent theoretical and simulation work, adding more physics (e.g., radiation pressure, ionisation) and overcoming previous limitations in numerical methods, have found that increasing the feedback, initial density fluctuations and turbulence leads to an increase in the fraction of the final stellar mass which comes directly from an initial gravitationally bound core (Krumholz et al. 2007, 2012; Hennebelle et al. 2011; Commercon, Hennebelle, \& Henning 2011; Myers

\footnotetext{
${ }^{1}$ Although see Izumi et al. (2014) who suggest a star system in the extreme outer galaxy ( $>18 \mathrm{kpc}$ ) may have been formed by large scale collision.
} 
et al. 2012; Bonnell et al. 2004; Peters et al. 2010). The apparent dichotomy between the competitive and core accretion models seems to be less extreme, or at least the reasons for the dichotomy are now better understood.

Nevertheless, understanding whether the two extreme formation scenarios are viable routes for high-mass stars to assemble their mass has important consequences for the host galaxy. If high-mass stars can only form in the presence of an attendant cluster, the stellar initial mass function (IMF) will be sampled very differently than if high-mass stars can (albeit rarely) form in isolation (Bastian et al. 2010). When averaged on galactic scales, this can make a dramatic difference in the number of (very) high-mass stars, and hence the level of feedback, chemical enrichment etc.

Given the wider importance of how the IMF is sampled, many observational studies have tried to find evidence of high-mass stars forming in isolation (Bressert et al. 2012; Tout et al. 1997; Weidner, Kroupa, \& Maschberger 2009; Weidner \& Kroupa 2005; Parker \& Goodwin 2007; de Wit et al. 2005; Schilbach \& Röser 2008). These previous searches have primarily focused on optical and infrared data to find young high-mass stars with no lower mass young stars around them. However, as these high-mass stars must already have cleared their natal gas cloud in order to be optically visible, it is very difficult to determine if they formed at their present location, as opposed to having been ejected from their parent stellar nursery of lower mass stars.

In this paper, we try a different approach, aiming to find very young high-mass stars while they are still embedded in their natal gas cloud. While extinction makes it impossible to find these objects in the optical and near-IR, their prodigious luminosity and Lyman continuum flux means they should be conspicuous at far-IR wavelengths and have bright $\mathrm{cm}$ continuum, free-free emission. Even in the most optimistic scenarios, high-mass stars forming in isolation are expected to be very rare, requiring large-area surveys to identify candidates. Thanks to an enormous effort from the Galactic observational community, Galactic plane surveys now exist across much of the electromagnetic spectrum at sufficient sensitivity and resolution to identify the majority of young high-mass star formation regions in the Galaxy. With the data now in hand, we aim to use a simple selection criterion to pick out the best candidates for young, high-mass stars forming in isolation.

\section{SAMPLE SELECTION}

For this first attempt to try and find examples of high-mass stars forming in isolation, we used a series of data summarised by Urquhart et al. (2013) for ATLASGAL Schuller et al. (2009) and CORNISH (Purcell et al. 2013). Urquhart et al. (2013) noted that targeted surveys of compact and ultracompact (UC) HII regions identified by infrared colours can be contaminated with intermediate mass young stellar objects (YSOs) and planetary nebulae (PNe). However, by incorpo-

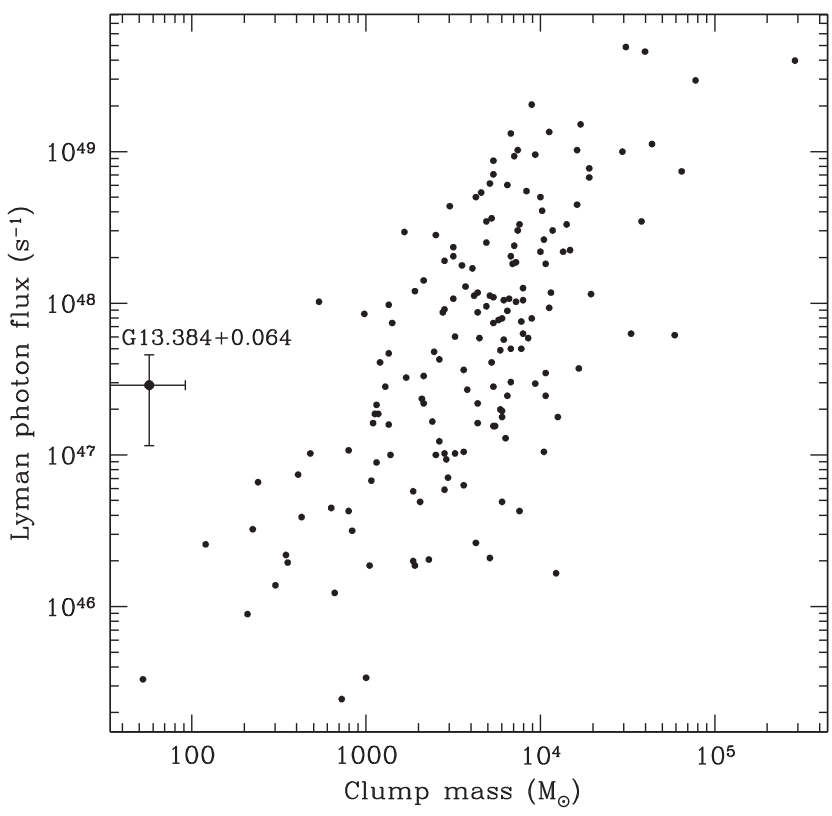

Figure 1. Plot of clump mass versus Lyman photon flux for a series of ATLASGAL and CORNISH sources, similar to the upper panel of Figure 26 in Urquhart et al. (2013). When the clump mass is plotted against the bolometric luminosity, the source at G13.384 + 0.064 does not stand out as different compared to other star-forming regions. This discrepancy is investigated in this paper. The error bars represent the best available data reported in this paper.

rating radio astronomy data mixed into the identification process, this is no longer a concern. Intermediate mass stars do not show radio continuum emission, so would be eliminated in the cross match. PNe are not usually associated with dust that is bright enough to be detected by ATLASGAL. ${ }^{2}$

We use their selection criteria to select approximately 200 bona fide YSOs, associated with compact and UC HII regions. Figure 1 compares the clump gas mass and Lyman continuum flux of these regions. The source G13.384 + 0.064 stands out in Figure 1 as having a very low gas mass for its Lyman continuum flux. This Figure is closely matched to the upper panel from Figure 26 in Urquhart et al. (2013). The aim of this paper is to better constrain the luminosity, mass, and lyman photon flux based on a thorough literature search of the region and using GLIMPSE (Churchwell et al. 2009; Benjamin et al. 2003) and MIPSGAL (Carey et al. 2009) data to to test for evidence of a surrounding cluster. We selected this as the most promising candidate in our initial sample for a high-mass star forming in isolation. Below we describe our efforts to use data in the literature to determine whether or not we can confirm or rule out this status.

\footnotetext{
${ }^{2}$ There were some instances noted where PNe were identified in ATLASGAL by their mid-infrared emission. However, they were removed from the source list and MonteCarlo simulations agreed with their identified numbers.
} 

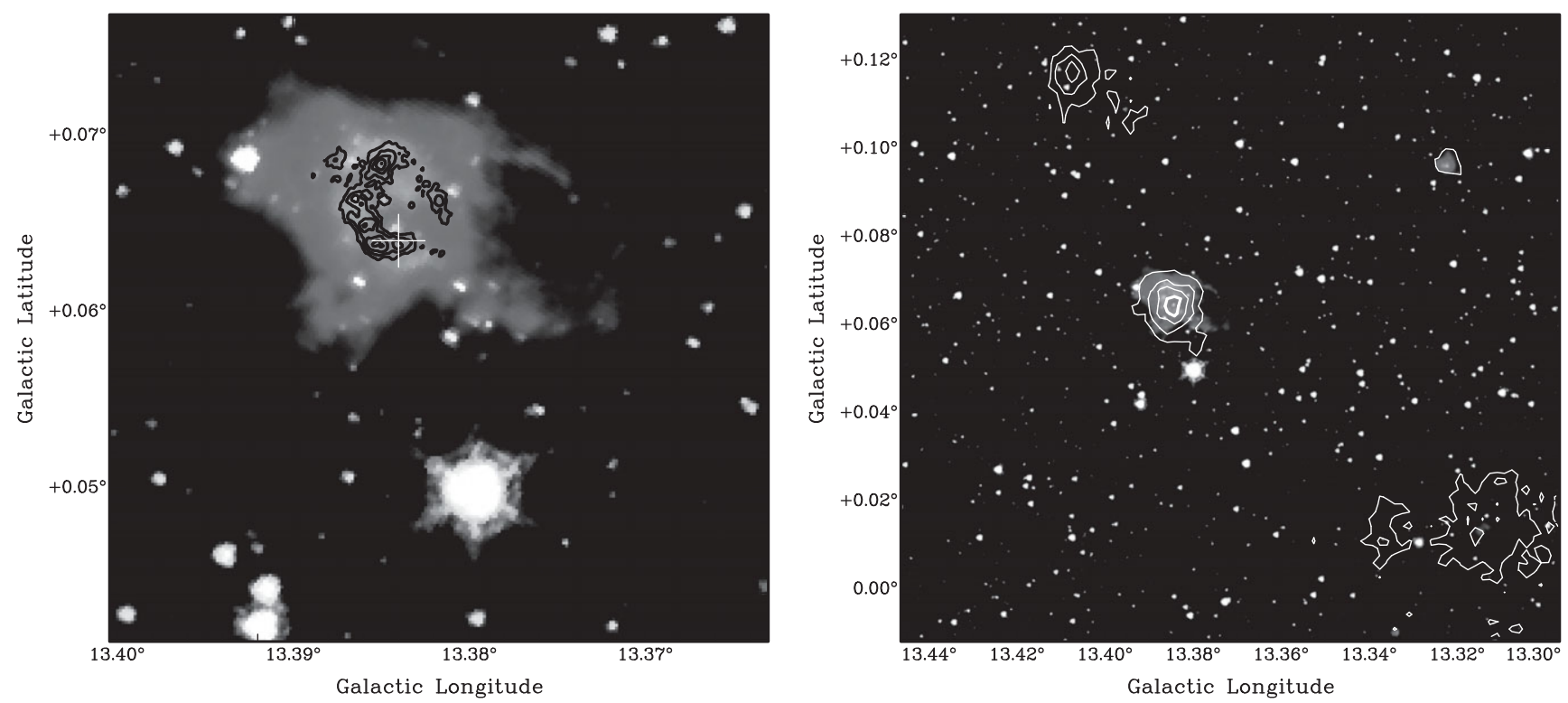

Figure 2. GLIMPSE 3 colour image with blue as $3.6 \mu \mathrm{m}$, green as $4.5 \mu \mathrm{m}$, and red as $8.0 \mu \mathrm{m}$. Contour image on the left shows CORNISH $5 \mathrm{GHz}$ radio continuum emission from $2 \mathrm{mJy} / \mathrm{beam}$ to $10 \mathrm{mJy} / \mathrm{beam}$ in steps of $2 \mathrm{mJy} / \mathrm{beam}$. The white cross on the image on the left represents the centre of the ATLASGAL contours and the image on the right shows the GLIMPSE 3 colour image with ATLASGAL contours. The contour levels are from $0.25 \mathrm{Jy} / \mathrm{beam}$ and increase in steps of $0.25 \mathrm{Jy} /$ beam up to $1.0 \mathrm{Jy} / \mathrm{beam}$. We identify an evolved star, seen at G13.380 + 0.050 and discussed in Section 3.8 that is unrelated to the source.

\section{RESULTS}

\subsection{Infrared \& radio observation}

The GLIMPSE (Churchwell et al. 2009; Benjamin et al. 2003) and MIPSGAL (Carey et al. 2009) images of the star-forming region G13.384 + 0.064 show a bright core surrounded by gas with an empty area or bubble between the two (Figure 2). The bubbles are similar to those seen in other high-mass star-forming regions and they are likely to be created by stellar feedback (e.g., Weaver, McCray, \& Castor 1977). The CORNISH radio continuum image has sufficient resolution to show an extended shell like source in the centre of the region (Figure 2). The region inside the radio emission also contains low levels of diffuse emission in the UKIRT Infrared Deep Sky Survey (UKIDSS) $K$-band images, as shown in Figure 4. It is likely this emission corresponds to Brackett Gamma (B $\gamma)$ emission associated with the HII region, as the morphology of the diffuse $K$-band emission resembles the radio continuum contours (Beck et al. 2010).

We used data from UKIDSS (Lawrence et al. 2007), 2MASS (Skrutskie et al. 2006) and GLIMPSE (Churchwell et al. 2009; Benjamin et al. 2003) to investigate the near and mid-IR source populations surrounding G13.384 + 0.064 and search for an embedded young stellar population. First, we downloaded all the sources in the UKIDSS point source catalogue within a 10 arc min radius of G13.384 + 0.064 and looked for an increase in the surface density of infrared objects towards the source. There is no statistical difference in the surface density of infrared sources at the location of G13.384 + 0.064 compared to similar regions close to, but offset from the ATLASGAL contours. We conclude there is no evidence of a cluster based on source density.

The three-colour diagram of UKIDSS data, Figure 4, shows some red stars within the contours from CORNISH. To determine if these are field stars behind the dust cloud or if they are within the cloud, we use the UKIDSS, 2MASS (for saturated UKIDSS sources), and GLIMPSE photometry data to plot a colour-colour diagram (CCD) and colour-magnitude diagrams (CMDs) of the infrared sources on and off G13.384 +0.064 . Figure 3 shows a representative CCD using $H$ and $K$-band data from UKIDDS (Lawrence et al. 2007) and 2MASS (Skrutskie et al. 2006) and $L(3.6 \mu \mathrm{m})$ band data from GLIMPSE (Churchwell et al. 2009; Benjamin et al. 2003) for a 30 arcsec radius around the source at G13.384 +0.064 (matching with the contours from ATLASGAL shown in Figure 2) and off the source at G13.36 + 0.075, as shown in Figure 3. The reddening vector was determined from Nishiyama et al. (2009) and the IMF data was plotted from Lejeune \& Schaerer (2001) for a cluster less than $10^{5}$ years old. While there are clearly reddened sources in the field, the CCD does not show an obvious excess of intrinsically red stars towards $\mathrm{G} 13.384+0.064$. The reddening value for each star was measured and a $K S$ test was completed to see if the two populations of data (on and off source) were similar. The results suggest that two populations could not be differentiated, making the red stars (from $K$ band UKIDSS data) in Figure 4 likely to be field stars and not representative of a cluster. The only possible exceptions are the two blue dots at $[\mathrm{K}]-[3.6] \sim 1.5$, which are potential candidates for YSOs of interest. However, without higher resolution data it is not possible to determine if these are simply highly 


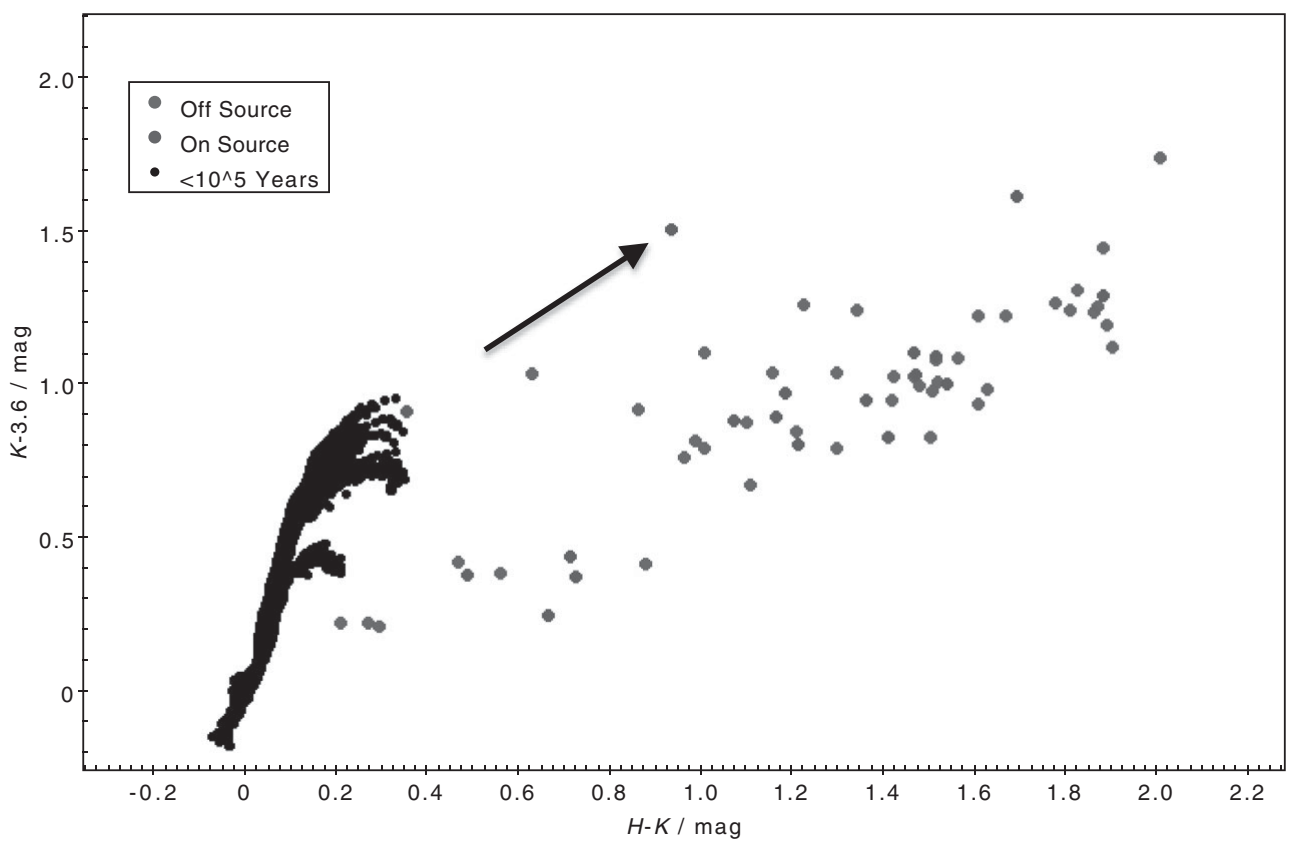

Figure 3. Colour-Colour diagram plotting sources 30 arcsec around G13.384 +0.064 (on source;blue) and 30 arcsec around G13.36 + 0.075 (off source;red). The IMF curve is data representing a cluster $<10^{5}$ years old from Lejeune \& Schaerer (2001). The vector represents the extinction vector plotted as per Nishiyama et al. (2009). There are no clear signs there are a cluster of main sequence stars.

extincted background stars or intrinsically red YSOs. Regardless of the nature of these two sources, the IR information from these three surveys data show no evidence of a sizeable distributed population of YSOs in the region.

We also note that these data may not be sensitive enough to detect stars deeply embedded into the natal cloud. As described in Feldt et al. (1998), it might be possible to hide a cluster of smaller stars. For further discussion, see Section 4.2.

\subsection{Distance}

The near kinematic distance of $1.9 \mathrm{kpc}$ and far distance of $14.1 \mathrm{kpc}$ was measured by Schlingman et al. (2011) and Shirley et al. (2013) using $\mathrm{HCO}^{+}(3-2)$ and $\mathrm{N}_{2} \mathrm{H}^{+}(3-2)$ observed by the Heinrich Hertz Submillimetre Telescope at $1.1 \mathrm{~mm}$. The velocity observed for these two molecules are $15.1 \mathrm{~km} \mathrm{~s}^{-1}$ for $\mathrm{HCO}^{+}$and $14.1 \mathrm{~km} \mathrm{~s}^{-1}$ for $\mathrm{N}_{2} \mathrm{H}^{+}$. Lockman (1989) reported $3 \mathrm{~cm}$ radio recombination line observations completed using the Greenbank telescope with an observed $V_{\text {LSR }}$ of $18.3 \pm 1.8 \mathrm{~km} \mathrm{~s}^{-1}$. However, Lockman (1989) commented that the FWHM line for this source was so broad that the results could not be easily interpreted.

$\mathrm{H}_{2} \mathrm{CO}$ was observed at $4.83 \mathrm{GHz}$, using the Nanshan station (Du et al. 2011). The results for the source G13.384 +0.064 show two molecular clouds. The first cloud has a velocity of $10.71 \pm 0.40 \mathrm{~km} \mathrm{~s}^{-1}$ and the second has a velocity of $51.34 \pm 0.28 \mathrm{~km} \mathrm{~s}^{-1}$. The velocity of the first cloud agrees well with the $\mathrm{HCO}^{+}, \mathrm{N}_{2} \mathrm{H}^{+}$, and radio recombination observations. Data from the James Clark Maxwell Telescope

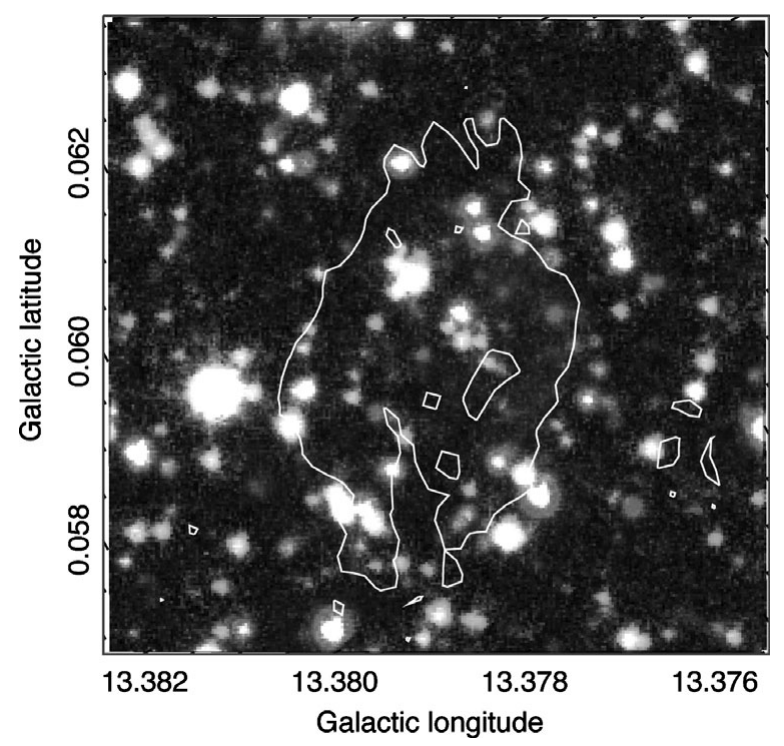

Figure 4. Three-colour image with red at $K$ band, green at $H$ band, and blue at $J$ band of UKIDSS data overlaid with contours from CORNISH $5 \mathrm{GHz}$ radio continuum emission with contours at 0.19 and $0.32 \mathrm{Jy} / \mathrm{beam}$.

(JCMT) reported three velocity components for ${ }^{12} \mathrm{CO}(\mathrm{J}=3-$ 2) at 10,15 , and $50 \mathrm{~km} \mathrm{~s}^{-1}$ (Dempsey et al. 2013). It is likely, then, that the star formation is associated with the gas at $15 \mathrm{~km} \mathrm{~s}^{-1}$, rather than the $50 \mathrm{~km} \mathrm{~s}^{-1}$, since the gas at $50 \mathrm{~km} \mathrm{~s}^{-1}$ only appears in the relatively low density gas tracers of $\mathrm{H}_{2} \mathrm{CO}$ and ${ }^{12} \mathrm{CO}$ and the star formation is most likely associated with the denser gas. 

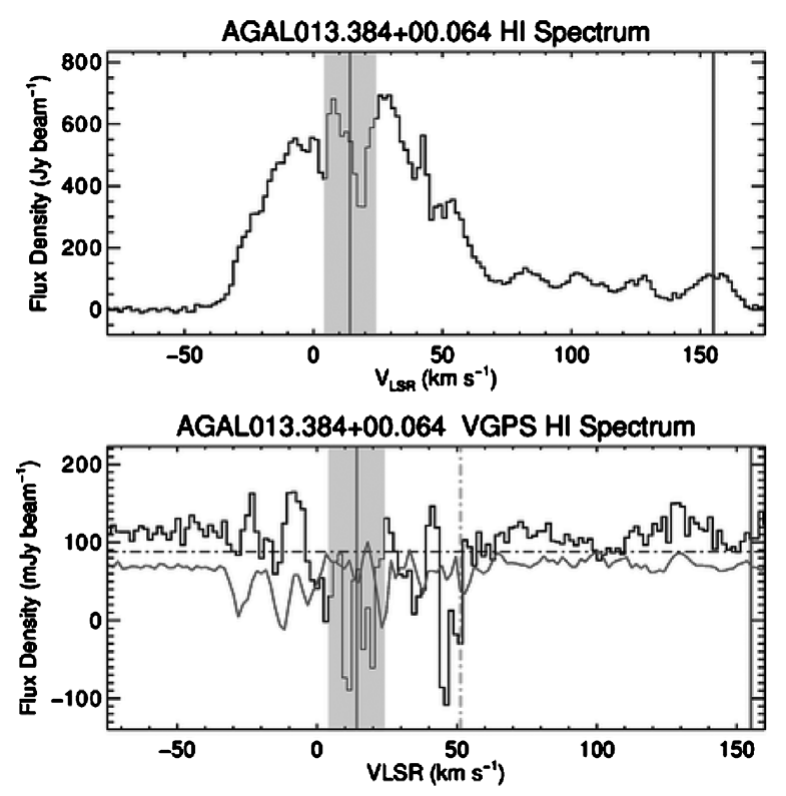

Figure 5. The HI continuum subtracted (top) and HI continuum (bottom) profiles seen towards G13.384 + 0.064 and its associated HII. In both of these panels, the source and tangent velocities are indicated by the red and blue vertical lines and the grey region shows a region $10 \mathrm{~km} \mathrm{~s}^{-1}$ either side of the source velocity. In the lower panel, the green line indicates the maximum velocity found of the absorption features and the magenta line shows the $5 \sigma$ rms noise for the HI data [see Urquhart et al. (2012) for more details]. The presence of an absorption feature at a similar velocity as the source in the upper panel and the lack of absorption features up to the tangent velocity in the lower panel both strongly support and near kinematic distance for this source.

The distance to this region has been determined by Schlingman et al. (2011) from the radial velocity and the Brand \& Blitz (1993) Galactic rotation curve. An issue that affects all sources located inside the solar circle is that there are two kinematic solutions spaced at equal distances from the tangent position; these are commonly referred to as the near and far distances. These distance ambiguities can be resolved using HI data by comparing the velocity of absorption dips seen in the spectra with the source velocity as measured from thermally excited molecular lines. The two most commonly used methods are HI self-absorption (HISA) (e.g., Jackson et al. 2002; Roman et al. 2009) and HI emissionabsorption (HIEA) (e.g., Kolpak et al. 2003; Andersen \& Bania 2009; Urquhart et al. 2012).

In Figure 5, we present the HISA profile (continuum subtracted) and HIEA (continuum included) HI profiles seen towards G13.384 +0.064 and its associated HII region. The source velocity is approximately coincident with the velocity of a broad absorption feature seen in the HI profile; this is consistent with the source being located at the near distance due to the fact that there is too much intervening warm HI gas at the same velocity as the source for any absorption to be present for sources located at the far distance.

The HIEA method is based on the principle that for any strong emission source located at the far distance we would

expect to observe absorption features at all velocities up to and including the tangent velocity. This is due to the high density of cold HI clouds along any line of sight through the inner Galaxy. The lack of any absorption features between 50 and $150 \mathrm{~km} \mathrm{~s}^{-1}$ would suggest that the source is again located at the near distance. The absorption seen at $50 \mathrm{~km} \mathrm{~s}^{-1}$ may suggest that the source velocity may be incorrectly assigned to this source and may in fact be associated with another object within the line of sight. However, the lack of any emission from high-density molecular tracers $\left(\mathrm{HCO}^{+}\right.$ and $\mathrm{N}_{2} \mathrm{H}^{+}$) would rule out this possibility. Both methods therefore suggest a near distance is more likely. For further discussion, see Section 4.1.

The uncertainties in the near kinematic distance, allowing for $\pm 10 \mathrm{~km} \mathrm{~s}^{-1}$ when the streaming motions and peculiar velocities are considered, is $\pm 0.8 \mathrm{kpc}$.

\subsection{Dust derived mass}

The near distance of $1.9 \pm 0.8 \mathrm{kpc}$ was used by Miettinen (2012) and Urquhart et al. (2013) to calculate the mass of the cloud surrounding the star as being $65 \pm 47$ and $105 \pm 73 \mathrm{M}_{\odot}$, respectively. Urquhart et al. (2013) used the integrated flux measured in ATLASGAL (Contreras et al. 2013) at $870 \mu \mathrm{m}$ and a temperature of $20 \mathrm{~K}$. Miettinen (2012) used $870 \mu \mathrm{m}$ observations from LABOCA and assumed a temperature of $35 \mathrm{~K}$. Since both reported masses are from the same instrument the differences are from the different temperatures and calibration errors. By using the Hildebrand (1983) equation and making the same assumption as stated in Equation (1) of Urquhart et al. (2013), we determine the cloud mass to a greater accuracy from the derived values specific to this source.

$$
\frac{M_{\text {clump }}}{M_{\odot}}=\left(\frac{D}{\mathrm{kpc}}\right)^{2}\left(\frac{S_{v}}{m J y}\right) \frac{R}{B_{v}\left(T_{\text {dust }}\right) \kappa_{v}},
$$

where $S_{v}$ is the integrated flux at $870 \mu \mathrm{m}$ of $603.94 \mathrm{mJy}$ (Schuller et al. 2009), $D$ is the heliocentric distance to the source ( $1.9 \pm 0.8 \mathrm{kpc}$; Section 3.2), $R$ is the dust-to-gas mass ratio (assumed to be 100), $B_{v}$ is the Planck function for a dust temperature $T_{\text {dust }}$ (calculated in Section 3.4 to be $33.7 \pm$ $1.5 \mathrm{~K}$ ), and $\kappa_{v}$ is the dust absorption coefficient taken as $1.85 \mathrm{~cm}^{2} \mathrm{~g}^{-1}$ (as used by Urquhart et al. 2013). This yields a value for the clump mass around the star as $57 \pm 35 \mathrm{M}_{\odot}$.

\subsection{Luminosity}

An estimation of the luminosity was determined from the IRAS fluxes (Neugebauer et al. 1984) using the equation initially published by Casoli et al. (1986) but using the same assumptions as Equation (3) of Walsh et al. (1997).

$$
F_{\text {tot }}=\left(f_{12} \delta v_{12}+f_{25} \delta v_{25}+f_{60} \delta v_{60}+f_{100} \delta v_{100}\right) / 0.61 \text {. }
$$

This yielded a value of $7.2 \times 10^{3} \mathrm{~L}_{\odot}$. This value is similar to that reported by Miettinen (2012) of $7.4 \times 10^{3} \mathrm{~L}_{\odot}$, which also uses the IRAS flux, but using the same assumptions 


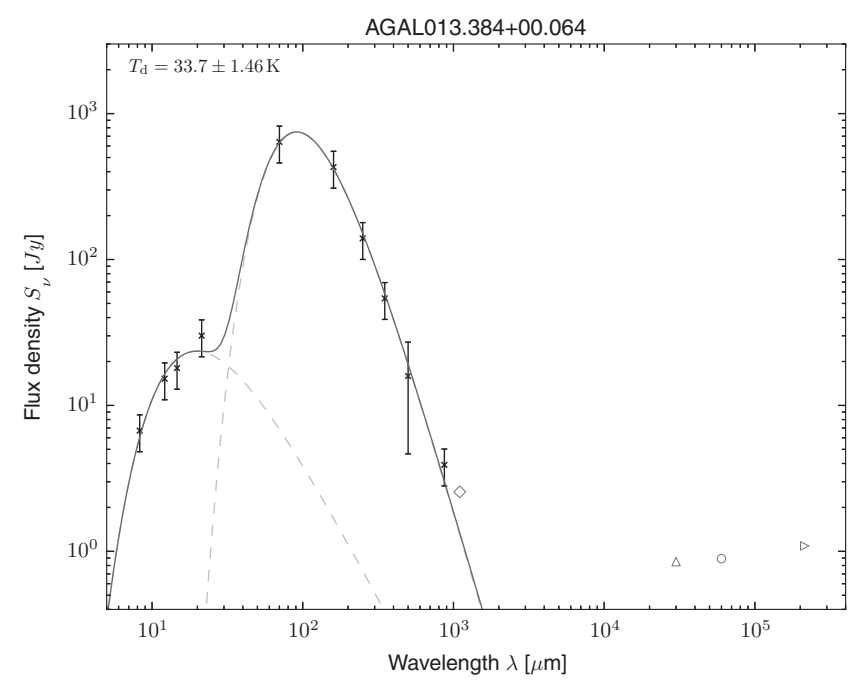

Figure 6. Spectral energy distribution created by data of different surveys plotted on a log scale. To obtain the dust temperature and luminosity, a two-component model was fitted to the flux densities measured through aperture photometry from the MSX (Price et al. 2001), Hi-GAL (Molinari et al. 2010), and ATLASGAL (Schuller et al. 2009) maps (blue fit). Additionally, catalogued data of the extended emission from the radio as well as some select infrared and submillimetre data. Open Triangle-MSX (Egan et al. 2003), Diamond-BOLOCAM (Schlingman et al. 2011), TriangleNobeyama (Handa et al. 1987), Open Circle-VLA 5 GHz (Becker et al. 1994), and Triangle Right-VLA 1.5 GHz (Garwood et al. 1988).

as Casoli et al. (1986). The bolometric luminosity reported by Urquhart et al. (2013), calculated by scaling the MSX $21 \mu \mathrm{m}$ flux, was $3.4 \times 10^{3} \mathrm{~L}_{\odot}$ which is almost half the value calculated from the IRAS fluxes. We note that the luminosity values reported by Miettinen (2012), Urquhart et al. (2013) and contained in this work, all assume a distance of $1.9 \mathrm{kpc}$.

The IRAS measurements can be considered as a strong upper limit on the bolometric luminosity. The large IRAS beam encapsulates all emission and there is no loss of flux due to extended emission that is masked out with other observations employing nodding or jittering method, such as BOLOCAM or ATLASGAL.

A more accurate value of the bolometric luminosity is measured using two component fitting of the spectral energy distribution (SED). The integrated flux data was compiled from a combination of reported catalogues values ranging from $4 \mu \mathrm{m}$ to $21 \mathrm{~cm}$ as well as measured values through aperture photometry from the MSX (Price et al. 2001), HiGAL (Molinari et al. 2010), and ATLASGAL (Schuller et al. 2009) maps. A plot of the integrated fluxes versus wavelength shows a curve that peaks in the IR and is flat at radio wavelengths as shown in Figure 6. This curve is typical of the SED of an embedded forming high-mass star. The peak in the infrared emission is characteristic of the short wavelength stellar light being reprocessed to longer wavelengths by dust in the surrounding gas cloud, and the flat region is from free-free radio emission characteristic of an HII region.
The flux density from the MSX, Hi-GAL, and ATLASGAL maps, Fapp, was measured within an aperture radius of 35.7 , corresponding to $3 \sigma$ of a Gaussian fitted to the source and centred on the peak flux pixel position of the $250 \mu \mathrm{m}$ image. The background flux density, Fbg, was obtained as the median pixel value from an annulus with $r_{\text {inner }}=47.6$ arcsec to $r_{\text {outer }}=59.5$ arcsec around the aperture. Subtracting the background flux density from the aperture flux density yields the background corrected source flux $F=F a p p-F b g$, reconstructing the SED in ten bands from $8 \mu \mathrm{m}$ to $870 \mu \mathrm{m}$. The errors of the flux densities are calculated by adding the absolute calibration uncertainty to the intrinsic measurement error in quadrature. We assume a measurement uncertainty of $20 \%$ for all bands except for the $500 \mu \mathrm{m}$ band, where we take into account the large pixel size of $15 \mathrm{arcsec}$, hence assuming a measurement uncertainty of $50 \%$.

The SED was then fitted with a two-component model consisting of a greybody and blackbody. The greybody (i.e., a modified blackbody) models the cold dust envelope's emission, taking into account the wavelength dependence of the dust in the far-infrared to submillimetre wavelength regime, whereas the blackbody models a hot, optically thick, deeply embedded component:

$$
F_{\lambda}\left(T_{\mathrm{d}}, \beta, \tau_{870}, T_{\mathrm{h}}, \Omega_{\mathrm{h}}\right)=F_{\lambda, \text { hot }}\left(T_{\mathrm{h}}, \Omega_{\mathrm{h}}\right)+F_{\lambda, \text { dust }}\left(T_{\mathrm{d}}, \tau_{870}\right),
$$

where $F_{\lambda \text {,hot }}$ is the hot component given by a blackbody scaled with the effective solid angle of the hot component and $F_{\lambda \text {,dust }}$ is the greybody emission from the dust envelope given by

$$
F_{\lambda, \text { dust }}\left(T_{\mathrm{d}}, \tau_{870}\right)=\Omega_{\mathrm{d}} \cdot B_{\lambda}\left(T_{\mathrm{d}}\right) \cdot\left(1-e^{-\tau_{870}\left(\frac{870 \mu \mathrm{m}}{\lambda}\right)^{\beta}}\right),
$$

where $\Omega_{\mathrm{d}}$ is the solid angle subtended by the source, $B_{\lambda}\left(T_{\mathrm{d}}\right)$ the blackbody intensity at the dust temperature $T_{\mathrm{d}}, \tau_{870}$ the dust optical depth at the reference wavelength of $870 \mu \mathrm{m}$ and $\beta$ the dust spectral index. We leave the dust spectral index $\beta$ fixed to a value of 1.75 , as computed as the mean value from the dust opacities of Ossenkopf \& Henning (1994) for the submillimetre regime. As a result the dust temperature was determined to be $33.7 \pm 1.5 \mathrm{~K}$, which is consistent with the result used by Miettinen (2012) and a luminosity of $4.1( \pm 1.7) \times 10^{3} \mathrm{~L}_{\odot}$ which is comparable to that reported by Urquhart et al. (2013) and is consistent with the upper limit from the IRAS measurements.

\subsection{Lyman continuum flux}

A number of observations of the region were completed in the radio using the National Radio Astronomy observatory Very Large Array (NRAOVLA) (Zoonematkermani et al. 1990; Becker et al. 1994; Garwood et al. 1988; Purcell et al. 2013), Nanshan Radio Telescope (Du et al. 2011), NRAO Green Bank (GBT) (Lockman 1989), and the Effelsberg 100'm Telescope (Alltenhoff, Downs, \& Pauls 1978). The integrated flux from two $6 \mathrm{~cm}$ observations, CORNISH (Purcell et al. 2013) and VLA 5 GHz Survey (Becker et al. 1994), were used to calculate the Lyman-continuum flux based on 
Equations (1) and (3) in Kurtz et al. (1994), which represent modified equations presented by Mezger \& Henderson (1967). Both of these surveys were completed using the NRAO VLA but CORNISH used B configuration,with a restoring beam of 1.5 arcsec, which is not as sensitive to the extended emission as the survey done by Becker et al. (1994) which was conducted in C configuration and has a restoring beam of 4 arcsec. Therefore, we expect the $\mathrm{C}$-array data to recover more emission. The logarithm of the number of Lyman continuum photons per second $\left(\log \mathrm{N}_{c}\right)$ using the CORNISH integrated flux of $603 \mathrm{mJy}$ and an assumed gas to dust ratio of 100 , was determined to be 47.3 photon s ${ }^{-1}$, as reported by Urquhart et al. (2013). Using the integrated flux from Becker et al. (1994) of $891.8 \pm 20.9 \mathrm{mJy}, \log \left(\mathrm{N}_{c}\right)=$ $47.3_{-0.4}^{+0.2}$ photon s ${ }^{-1}$.

The flux derived by Altenhoff et al. (1978) for the $100 \mathrm{~m}$ Effelsberg telescope at $4.9 \mathrm{GHz}$ is $0.9 \mathrm{Jy}$, which is similar to the value reported by Becker et al. (1994) of $0.891 \mathrm{Jy}$. This suggests that all the extended emission was accounted for in the $\mathrm{C}$ configuration observations.

\subsection{Star type}

The Lyman-continuum flux and bolometric luminosity are compared to Table 1 in Davies et al. (2011) to determine a mass of the star powering the HII region. The bolometric luminosity as determined by the SED fit is $\log \left(\mathrm{L}_{\star} / \mathrm{L}_{\odot}\right)=$ $3.61 \pm 0.14$. This relates to a star between $9-12 \mathrm{M}_{\odot}$. The $\log$ of the Lyman photon flux is $47.3_{-0.4}^{+0.2}$ photon $\mathrm{s}^{-1}$. This relates to a star between $15-20 \mathrm{M}_{\odot}$.

The mass measured using the SED fit is $13 \pm 6 \mathrm{M}_{\odot}$. A main sequence star type $B$, is a star with a mass of 2.1$16 \mathrm{M}_{\odot}$ which is consistent with the values measured for this source. As $B$ stars are known to have an excess of Lyman flux (see Section 4.1), the star is likely to be between 9-12 $\mathrm{M}_{\odot}$ as suggested by the bolometric luminosity measurement [as suggested by Table 1 in Davies et al. (2011) and SED curve in Figure 6].

\subsection{An unrelated evolved star}

Figure 2 shows a bright infrared star at G13.380 + 0.050, approximately 1 arcmin to the south. This star coincides with a $1612 \mathrm{MHz} \mathrm{OH}$ maser, detected by Sevenster et al. (2001), indicating that the star is evolved and unlikely to be part of the same star formation process. Given the offset between this star and G13.384 +0.064, the projected distance is $0.5 \mathrm{pc}$. The image in Figure 2 shows the structure of the cloud in the infrared extends toward the star at G13.380 + 0.050 but does not overlap with this star. Furthermore, there is no edge brightening in the extended infrared emission observed. This suggests that the bright star is unlikely to have a direct physical influence on the star-forming region and is most likely an unrelated star projected along the line of sight, but not necessarily physically close to the star-forming region.

\section{DISCUSSION}

\subsection{Far distance consideration}

The radio continuum-derived luminosity of $3.6 \times 10^{4} \mathrm{~L}_{\odot}$ derived in Section 3.5 is a factor of ten greater than the dustderived luminosity $4.1 \times 10^{3} \mathrm{~L}_{\odot}$. We must reconcile this difference.

If we assume that $\mathrm{G} 13.384+0.064$ is at the far kinematic distance of $14.1 \mathrm{kpc}$, then the radio-derived and dustderived luminosities are $3.2 \times 10^{5} \mathrm{~L}_{\odot}$ and $2.2 \times 10^{5} \mathrm{~L}_{\odot}$, respectively. These two luminosities are more consistent than those when the near distance is assumed. However, even though this luminosity argument favours the far kinematic distance, we still favour the near kinematic distance for the following reasons.

First, as discussed in Section 3.2, the HI observations strongly favour the near distance. The HI observations analysed by Urquhart et al. (2013) and shown in Figure 5 shows little evidence of high velocity HI self-absorption, which would be expected from intervening gas, if it was at the far kinematic distance. The only high velocity absorption is seen around $50 \mathrm{~km} \mathrm{~s}^{-1}$. This corresponds to the velocity of the cloud seen in the $\mathrm{H}_{2} \mathrm{CO}$ and ${ }^{12} \mathrm{CO}$ observations reported previously and so the absorption is most likely not related to $\mathrm{G} 13.384+0.064$.

Second, there are uncertainties in the expected Lyman continuum flux from young high-mass stars. Smith (2014) concludes that the Lyman continuum flux of early B type stars may be in excess of previous models, caused by the accretion of cold gas from the circumstellar disk onto hot-spots on the surface of the young star. Comparing the measurements for G13.384 + 0.064, we find that it sits on the upper edge of their model such that the excess radio continuum flux is consistent with the bolometric luminosity. If the radio continuum flux were much larger, this source would show an unaccounted for excess of radio continuum, compared to the dust-derived luminosity.

A final consideration is the expected number of lower mass stars surrounding the high-mass star if it were at the near vs. far kinematic distance. If G13.384 + 0.064 was at the far distance of $14.1 \mathrm{kpc}$, then the mass of the star would be approximately $38 \mathrm{M}_{\odot}$, based on the mass to luminosity relationship. The mass of the surrounding gas cloud would increase to $5800 \mathrm{M}_{\odot}$, making it very likely that a cluster is present. However, as discussed in Section 3.1, no evidence for a cluster is seen in the infrared images.

\subsection{Isolated vs. clustered star formation in G13.384 $+0.064$}

We now seek to use the properties derived above to determine whether G13.384 + 0.064 is indeed a high-mass star forming in isolation.

Parker \& Goodwin (2007) defines an isolated $B$ type star as a star $10 \mathrm{M}_{\odot}<\mathrm{M}_{*}<17.5 \mathrm{M}_{\odot}$ in which the cluster mass 
is $<100 \mathrm{M}_{\odot}$ and there are no $O$-stars present. The estimated mass of the star based on the SED curve is $13 \pm 6 \mathrm{M}_{\odot}$ and the estimated remaining clump mass is $57 \pm 35 \mathrm{M}_{\odot}$. Both of these are consistent with the definition described by Parker $\&$ Goodwin (2007) for isolated high-mass star formation.

Based solely on the detection of a strong radio continuum source, we can conclude that there must be at least one embedded high-mass star. Incorporating a model by Smith (2014), we find that such a star must account for nearly all (if not, all) of the infrared flux, leaving little room for a cluster. This is because lower mass stars will not contribute significantly to the radio-derived luminosity, but will significantly contribute to the infrared or dust-derived luminosity.

We can model what a cluster might look like, by constraining the quantities of dust-derived luminosity and Lyman continuum flux. In order to do this, we use a Monte-Carlo simulation of the IMF to generate a cluster. Based on previous work by Walsh et al. (2001), we found that commonly used functions of the IMF do not greatly affect the make-up of simulated clusters, so we choose the IMF model of Kroupa et al. (1993). In our simulation, we randomly generate stars between masses of 0.1 and $100 \mathrm{M}_{\odot}$, according to this IMF and then calculate the cluster physical parameters, such as total mass, luminosity, and Lyman continuum flux. In order to measure the relationship between Lyman continuum flux and luminosity, we use the values given in Table 1 of Thompson (1984) but we note that previous studies have found an excess of Lyman continuum photons from early $B$-type stars (eg., Urquhart et al. 2013). Therefore, in order to take into account the work of Smith (2014), we apply their most extreme case for ratio of Lyman flux, compared to previous models, where the Lyman flux may be reduced by up to an order of magnitude for the same star with the same bolometric luminosity (i.e., reduction of $10^{47.3}$ to $10^{46.3}$ photon s ${ }^{-1}$ ). It is important to note that in taking such an extreme reduction in Lyman flux, our simulation will favour the formation of a cluster, rather than an isolated star. This allows us to use the data of Thompson (1984) and correct it for more recent modelling by Smith (2014).

In our Monte-Carlo simulation, we continue to add members to the cluster until the total cluster luminosity is greater than $4.1 \times 10^{3} \mathrm{~L}_{\odot}$. We generated 68877 clusters with sufficient luminosity to meet this criterion. However, we note that the majority of generated clusters have total luminosities far in excess of this value. This is because the last star added to the cluster is typically a high-mass star with very high luminosity. Thus, we exclude those generated clusters that have luminosities in excess of the IRAS-derived luminosity $\left(7.2 \times 10^{3} \mathrm{~L}_{\odot}\right)$, leaving 30601 clusters. We choose the IRAS-derived luminosity here because it is a strong upper limit on the bolometric luminosity, given that IRAS will likely overestimate the total infrared flux, but not underestimate it.

Of our remaining clusters, we find that the median luminosity for the highest mass star in each cluster is $2.8 \times 10^{3} \mathrm{~L}_{\odot}$ which means that for most clusters, the luminosity is dom- inated by one star. We also find that the highest mass star generated in any cluster has a luminosity of $7.1 \times 10^{3} \mathrm{~L}_{\odot}$, which we calculate has a corresponding Lyman continuum flux of $10^{45.7}$ photons $\mathrm{s}^{-1}$. This Lyman flux is lower than we expect $\left(10^{46.3}\right.$ photons $\left.\mathrm{s}^{-1}\right)$ by about an order of magnitude. In summary, our simulations indicate that it is very difficult to randomly generate a cluster with the properties that we observe for G13.384 + 0.064. The only way to generate a good match is for the first star selected from the IMF to be a high-mass star with the right luminosity and Lyman flux properties. However, classifying such a single star as a cluster is questionable.

\section{CONCLUSIONS}

In this paper, we compared the Lyman-continuum photon flux and clump mass of approximately 200 star-forming regions to search for high-mass stars forming in isolation. We identified one source, G13.384 + 0.064, as a very promising candidate. Analysis completed in both the infrared and the radio, combined with Monte-Carlo modelling, shows this source is consistent with a single high-mass star in formation, and there is no strong evidence of a cluster. This verifies the choice of using Lyman flux and bolometric luminosity to identify such candidates. While the observations in the literature allowed us to rule out a large population of embedded young stars, further high-resolution, deep, infrared, and submillimetre observations are required to quantify just how small this population is, and thereby unambiguously determine if G13.384 + 0.064 is a high-mass star forming in isolation.

\section{ACKNOWLEDGEMENTS}

The authors would like to thank the referee for the comments and advice. SNL would like to thank Andy Longmore for very helpful discussions about the analysis of the infrared data. This research has made use of the SIMBAD database, operated at CDS, Strasbourg, France. This research has also made use of the VizieR catalogue access tool, CDS, Strasbourg, France. The original description of the VizieR service was published in A\&AS 143, 23.

\section{REFERENCES}

Altenhoff, W. J., Downs, D., \& Pauls, T. 1978, AAS, 35, 23

Andersen, L., \& Bania, T. 2009, ApJ, 690, 706

Bastian, N., et al. 2010, ARA\&A, 48, 339

Beck, T., et al. 2010, ApJ, 722, 1360

Becker, R. H., et al. 1994, A\&AS, 91, 347, 387

Benjamin, R., et al. 2003, PASP, 115, 953

Bressert, E., et al. 2012, A\&A, 542, 14

Bonnell, I., Bate, M., \& Zinnecker, H. 1998, MNRAS, 298, 93

Bonnell, I., et al. 2001, MNRAS, 324, 573

Bonnell, I., et al. 2004, MNRAS, 349, 735

Bonnell, I., \& Bate, M. 2006, MNRAS, 370, 488

Brand, J., \& Blitz, L. 1993, AAP, 275, 67

Carey, S. J., et al. 2009, ASP, 121, 875, 76

Casoli, et al. 1986, AA, 169, 281 
Churchwell, E., et al. 2009, PASP, 121, 213

Commercon, B., Hennebelle, P., \& Henning, T. 2011, ApJL, $742,1,6$

Contreras, Y., et al. 2013, JA\&A, 549, 45

Cutri, R. M., et al.2012, WISE Catalogues, 2311

Davies, B., et al. 2011, MNRAS, 416, 972

Dempsey, J. T., et al.2013, ApJS 209, 8

de Wit, W. J., et al. 2005, A\&A, 437, 247

Du, et al. 2011, A\&A, 532A, 127

Egan, M., Price, S., \& Kraemer, K. BAAS, 35, 1301

Feldt, et al. 1998, A\&A, 339, 759

Garwood, R., et al. 1988, ApJ, 96, 1655

Handa, J., et al. 1987, PASJ, 39, 709

Hennebelle, P., et al. 2011, A\&A, 528, 17

Hildebrand, R. H. 1983, QJRAS, 24, 267

Izumi, N., et al. 2014, ApJ, 795, 1, 66

Jackson, et al. 2002, ApJL, 566, L81

Kolpak, et al. 2003, ApJ, 582, 756

Kroupa, P., et al. 1993, MNRAS, 262, 545

Krumholz, et al. 2007, ApJ, 656, 2, 59

Krumholz, et al. 2012, ApJ, 760, 2, 155

Kurtz, S., et al. 1994, ApJS, 91, 659

Lawrence, et al. 2007, MNRAS, 379, 1599

Lockman, F. J. 1989, ApJS, 71, 469

Lejeune, \& Schaere 2001, A\&A, 366, 538

McKee, C., \& Tan, J., 2003, AJ, 585, 2, 850

Mezger, P. G., \& Henderson, A. P. 1967, ApJ, 147, 471

Miettinen, O. 2012, A\&A, 542, A101

Molinari, et al. 2010, PASP, 122, 314

Myers, A., et al. 2012, ApJ, 766, 2, 18
Neugebauer, G., et al. 1984, ApJL, 278, L1

Nishiyama, et al. 2009, ApJ, 696, 2, 1407

Ossenkopf, V., \& Henning, T. 1994, AAP, 291, 943

Parker, R., \& Goodwin, S. 2007, MNRAS, 380, 3, 1271

Peters, T., et al. 2010, ApJ, 711, 2, 1017

Price, S., et al. 2001, AJ, 121, 2819

Purcell, C. R., et al. 2013, ApJS, 205, 1

Roman, et al. 2009, ApJ, 699, 1153

Schilbach, E., \& Röser, S. 2008, A\&A, 489, 105

Schlingman, W., et al. 2011, ApJS 195, 2, 14

Schuller, F., et al. 2009, A\&A, 504, 415

Sevenster, M. N., et al. 2001, A\&A, 366, 481

Shirley, Y. L., et al. 2013, ApJS 209, 2

Smith, M. 2014, MNRAS, 438, 2, 1055

Skrutskie, M. F., et al. 2006, AJ, 131, 1163

Tan, J. C., et al. 2014, Protostars and Planets VI, 149

Thompson, R. I. 1984, ApJ, 283, 165

Tout, C. A., Aarseth, S. J., Pols, O. R., \& Eggleton, P. P. 1997, MNRAS, 291, 732

Urquhart, J. S., et al. 2012, MNRAS, 420, 2, 1656

Urquhart, J. S., et al. 2013, MNRAS, 435, 1, 400

Walsh, A. J., et al. 1997, MNRAS, 291, 261

Walsh, A. J., et al. 2001, MNRAS, 326, 1, 36

Weaver, R., McCray, R., \& Castor, J. 1977, ApJ, 218, 377

Weidner, C., \& Kroupa, P. 2005, ApJ, 625, 754

Weidner, C., Kroupa, P., \& Maschberger, T. 2009, MNRAS, 393, 663

Zinnecker, H., \& York, H. 2007, ARA\&A, 45, 1, 481

Zoonematkermani, S., et al. 1990, ApJS, 74, 181

Zuckerman, B., \& Dyck, H. M. 1986, ApJ, 311, 345 